\title{
Single institution experience on cancer among adolescents 15-19 years of age
}

\author{
Begül Yağcı-Küpeli, Canan Akyüz, Bilgehan Yalçın, Ali Varan, Tezer Kutluk, Münevver \\ Büyükpamukçu \\ Department of Pediatric Oncology, Hacettepe University Institute of Oncology, Ankara, Turkey. \\ E-mail:drbegul@yahoo.com \\ Received: 22nd March 2016, Revised 21st February 2017, Accepted: 6th April 2017
}

SUMMARY: Yağcı-Küpeli B, Akyüz C, Yalçın B, Varan A, Kutluk T, Büyükpamukçu M. Single institution experience on cancer among adolescents 15-19 years of age. Turk J Pediatr 2017; 59: 1-5.

Adolescent cancers differ from other age groups in terms of cancer types, treatment and outcome. We aimed to present our institutional data on survival of certain types of cancer in adolescents.

Hospital files were retrospectively evaluated for distribution of tumor types, clinical features, and overall and event-free survival (OS and EFS) rates in adolescents with malignant tumors.

Two hundred ninety-three cases between 15-19 years who were diagnosed with malignant tumor at our department in the last 38 years were included in the study. Mean age was 15.3 and median age was 15 years at time of diagnosis. Male/female ratio was found to be $1.8 / 1$. The most common cancers were non-Hodgkin lymphoma (NHL) (20.5\%), Hodgkin's lymphoma (HL) (19.8\%), central nervous system (CNS) tumors (10.9\%), osteosarcoma (10.6\%), Ewing sarcoma/primitive neuroectodermal tumors (EWN/PNET (9.9\%) nasopharyngeal carcinoma (NPC) $(9.6 \%)$. Non-compliance to medical treatment was observed in $10.9 \%$ of cases with significantly high noncompliance in NHL and osteosarcoma $(\mathrm{p}=0.02)$. Overall survival was $57.6 \%$ and EFS was $45.7 \%$ at a median follow-up time of 8.75 years.

In adolescents, lower OS and EFS rates than the younger age group are observed. The most appropriate management plan should be made according to the physical and psychosocial needs of patients in this age group.

Key words: adolescent, lymphoma, CNS tumors, nasopharyngeal carcinoma, outcome.

Adolescence can be defined as a transition period between childhood and adulthood. However, there is not a well-defined age range for this period. World Health Organization defines an individual between 10-19 years as an adolescent. From the oncologic point of view, adolescent cancers are defined as cancers seen between 15-19 years ${ }^{1-3}$. Lymphomas, leukemias, bone and soft tissue sarcomas, germ cell tumors, and epithelial tumors are the most common cancer types and embryonal tumors of childhood are rare. Despite significant improvement in cancer diagnosis, treatment and survival, similar improvement could not be observed in adolescent cancers. Furthermore, adolescents face many problems while dealing with cancer due to limited awareness about adolescents' requirements in oncology centers specific for children or adults. Less enrollment to clinical trials is another important issue that may be attributed to lower survival rates than other age groups ${ }^{4-7}$.

\section{Material and Methods}

Files of patients between 15-19 years of age diagnosed with cancer between 1970 and 2008 in Hacettepe University, Department of Pediatric Oncology were retrospectively reviewed. Demographic features of patients, cancer types and histopathological subgroups, primary cancer localizations, treatment modalities and non-compliance to treatment 
were analyzed using descriptive analysis.

All statistical analyses were performed using SPSS statistical software (SPSS Inc, Chicago, IL). Descriptive data are given as percentages. The Kaplan-Meier method was used for survival analysis. Overall survival (OS) was calculated from diagnosis to death or last visit. Event-free survival (EFS) was calculated from diagnosis to death, progression of the disease, or recurrence, whichever came first, or the last visit. Comparison between the patient groups was made by log-rank test. A $p$ value of $<0.05$ was considered statistically significant.

\section{Results}

Two hundred ninety-three adolescents with cancer were included in the study. There were
189 males and 104 females with a median age of 15 years. Median follow-up time was 8 years and 9 months (range: 1 year- 30 years). Lymphomas were the most common tumor in our group. Details of patients are given in Table I.

Male preponderance was observed in all tumor groups except for osteosarcoma. A significant male predominance was seen in Hodgkin and Non-Hodgkin lymphomas with male female ratios of $3.1: 1,3: 1$, respectively. The most common tumor locations were head and neck in lymphomas, extremities and pelvis in Ewing sarcoma/primitive neuroectodermal tumors (EWN/PNET), distal femur in osteosarcoma, and supratentorial region in central nervous system (CNS) tumors. Early stage (stage I or

Table I. General Characteristics of Patients

\begin{tabular}{lcc}
\hline & $\mathrm{N}$ & $\%$ \\
\hline Male & 189 & 64.5 \\
Female & 104 & 35.5 \\
Cancer types & & \\
NHL & 60 & 20.5 \\
Hodgkin's Lymphoma & 58 & 19.8 \\
CNS tumors & 32 & 10.9 \\
Osteosarcoma & 31 & 10.2 \\
EWN/PNET & 29 & 9,9 \\
NPC & 28 & 9.6 \\
Rhabdomyosarcoma and other STS & 9 & 3.1 \\
Germ cell tumors & 6 & 2.1 \\
Papillary thyroid carcinoma & 5 & 1.7 \\
GIS tumors & 5 & 1.7 \\
Renal cell carcinoma & 4 & 1.4 \\
Neuroblastoma & 4 & 1.4 \\
Langerhans cell histiocytosis & 3 & 1.1 \\
Other tumors & 19 & 6.6 \\
Treatment & & \\
Chemotherapy+radiotherapy & 147 & 50.2 \\
Chemotherapy & 49 & 16.7 \\
Chemotherapy+radiotherapy+surgery & 39 & 13.3 \\
Chemotherapy+surgery & 27 & 9.2 \\
Surgery & 15 & 5.1 \\
Surgery+Radiotherapy & 13 & 4.4 \\
Radiotherapy & 3 & 1.1 \\
Stage & & 60.4 \\
Early stage (I,II)/Non-metastatic & 177 & 39.6 \\
Advanced stage/Metastatic & 116 & \\
\hline NH Non-Hodin lymo; CNS: centra & 59 & \\
\hline
\end{tabular}

NHL: Non-Hodgkin lymphoma; CNS: central nervous system; EWN/PNET: Ewing sarcoma-primitive neuroectodermal tumor family; NPC: nasopharyngeal carcinoma; GIS: gastrointestinal system 
II) or localized disease was detected in $60.4 \%$ of cases and late stage (stage III or IV) or metastatic disease was detected in $39.6 \%$ of patients. Majority of patients with osteosarcoma $(74.5 \%)$ were metastatic at time of diagnosis. All patients with CNS tumors had localized disease and none of them have intracranial or spinal seeding.

The most frequent treatment combination was chemotherapy and radiotherapy. Irregular attendance to chemotherapy schedule and refusing therapy were defined as non-compliance to treatment. Percentage of non-compliance to treatment was 10.9 in whole group and higher in males than females. However, this difference was not statistically significant $(p=0.7)$. Noncompliance to treatment was more common in patients with NHL and osteosarcoma than other tumors. The difference according to non-compliance to treatment between tumor groups was statistically significant $(p=0.02)$. No significant difference was detected according to non-compliance to treatment between early stage or metastatic disease.

Overall survival (OS) was $57.6 \%$, event-free survival (EFS) was $45.7 \%$ (median follow-up time: 8 years and 9 months) (Fig. 1), five-year OS was $62.6 \%$, five-year EFS was $51.8 \%$ in whole group. Five-year OS rates were found to be $71 \%$ in CNS tumors, $56.2 \%$ in NHL, $85 \%$ in $\mathrm{HL}, 36.8 \%$ in EWN/PNET, $57.5 \%$ in NPC, $33.5 \%$ in osteosarcoma, $75.8 \%$ in other tumors. Statistically significant difference was detected according to five-year OS and EFS between cancer types $(p<0.001)$. Overall survival and EFS were better in patients with localized or early stage disease than patients with metastatic or late stage disease $(\mathrm{p}<0.001)$. No statistically significant difference was observed in OS and EFS rates according to compliance to treatment.

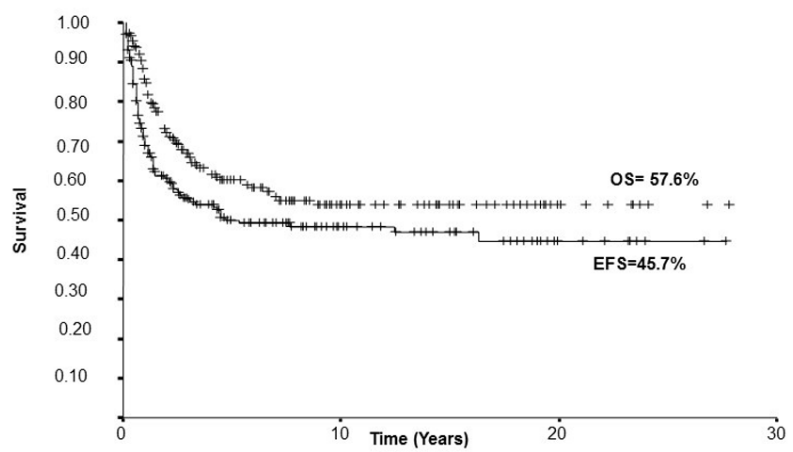

Fig. 1. Overall (OS) and event-free survival (EFS) of patients

\section{Discussion}

In oncology practice, cancers observed between 15 and 19 years are regarded as adolescent period cancers. Due to lack of standard age range, variable cancer incidences have been reported in literature ${ }^{1-9}$. In United States, annual cancer incidence between 15-19 years was reported to be 20 in $100,000^{10}$.

Adolescent cancers portray different characteristics compared to childhood and adult cancers $1,2,10,11$. The most common cancer types in this age group are leukemia, lymphoma, bone and soft tissue sarcomas, germ cell tumors, and epithelial tumors. In our study, leukemias were not included to our study and lymphomas, CNS tumors and bone and soft tissue sarcomas were the most common tumors. Germ cell tumors and epithelial tumors were not as frequent as expected. Possibly, this was because these types of adolescent cancers were treated in adult oncology centers. Other studies have also shown cancers like germ cell tumors, carcinomas and malignant melanomas were more likely to be treated in adult centers ${ }^{12,13}$.

Cancer was reported to be fourth common cause of death among adolescents in United States. Mortality in adolescent cancers arises from four major cancers: sarcomas, leukemia/ lymphomas, malignant CNS tumors and germ cell tumors ${ }^{11}$. Although an increase of $1.5 \%$ (per year) in 5-year overall survival in childhood cancers was observed during the last 25 years in America, only $0.9 \%$ increase (per year) was reported in adolescent survival rates $^{14}$. One of the main reasons for this consequence is lower clinical trial enrollment among adolescents. Furthermore, controversy still exists on treating an adolescent with cancer whether in a pediatric or adult oncology center ${ }^{15}$. Evidence suggests that better outcome is observed when adolescents with certain types of cancer are treated with pediatric protocols 16-19. A new concept of adolescent and young adult (AYA) oncology has emerged in the last decade. This new discipline, involved in patients from 15 to 29 (or 39) years of age, was created by cooperative study of pediatric and adult oncologists who use patient-focused multidisciplinary approach in management of cancer treatment ${ }^{20,21}$. Although this program has been constituted in Europe, North America and Australia, a discipline of AYA oncology is 
lacking in Turkey.

Five-year OS rates in adolescent cancers were reported to be $69 \%$ between years $1975-1984$ and $77 \%$ between 1985-1994 in United States ${ }^{10}$. European data revealed that OS in adolescent cancers were $73 \%$ in between 1988-1997 with geographical variations observed in northern and eastern parts of Europe ${ }^{22}$. 5-year overall survival was $62.6 \%$ in our study. Our OS rates were lower compared to American or European data in all cancer types. However, close OS rates to developed countries' results in certain cancer types such as Hodgkin lymphoma and malignant CNS tumors were observed in our study. Pediatric oncology concept had emerged during late 1970s in our country and a few pediatric oncology centers had been established in that time. Lower survival rates may be associated with limited resources.

Another possible factor for lower survival rates in adolescents with cancer is noncompliance to treatment. Studies have shown that adolescents have more problems than their younger and older counterparts in terms of compliance 23,24 . However, lack of precise definition of non-compliance, assessment and measurement can be difficult ${ }^{25}$. Direct evaluation through urine and serum samples and indirect assessment by interviewing with family members or health professionals can be used ${ }^{24}$. Non-compliance can be observed as delayed attending to chemotherapy courses or as not-using recommended medications as well as not attending long-term follow-up ${ }^{26}$. Complicated treatment regimens, unsuitable conditions of hospitals for adolescent's needs, desire of adolescent for independence, insufficient communication between adolescent and health care provider, denial of the disease by adolescent, feeling undefeatable against cancer observed in adolescents may be among factors leading to non-compliance ${ }^{11}$. Our study's retrospective design could not provide a thorough assessment for non-compliance. However, it was observed in one of ten adolescents with cancer. Patients with NHL and osteosarcoma were shown to be more non-compliant to treatment compared to other cancer types.

In conclusion, there are still many issues that need to be addressed in adolescent cancers even in developed countries. First of all, an attempt should be made for increasing the awareness about adolescent cancers in public to prevent the delay in diagnosis. Second, health care providers should be informed about characteristics of adolescent period in order to cope well with adolescents. Third, a suitable treatment center that meets the special needs of this age group provided for optimal treatment. Finally, enrollment to clinical trials of adolescents with cancer should be encouraged for a better outcome. Furthermore, the concept of AYA oncology discipline should be constituted even in developing countries.

\section{REFERENCES}

1. Evans M. Interacting with teenagers with cancer. In: Selby P, Bailey C, eds. Cancer and the Adolescent. London: BMJ Publishing Group, 1996: 251-263.

2. Whyte F, Smith L. A literature review of adolescence and cancer. Eur J Cancer Care 1997; 6: 137-146.

3. Oeffinger KC, Eshelman DA, Tomlinson GE, Buchanan GR. Programs for adult survivors of childhood cancer. J Clin Oncol 1998; 16: 2864-2867.

4. Leonard R, Coleman R, Gregor A, Regan J. Special problems of adolescents with cancer. J Cancer Care 1995; 5: 117-120.

5. Fritschi L, Coates M, McCredie M. Incidence of cancer among New South Wales adolescents: which classification scheme describes adolescent cancers better. Int J Cancer 1995; 60: 355-360.

6. Steliarova-Foucher E, Stiller C, Lacour B, Kaatsch P. International Classification of Childhood Cancer, third edition. Cancer 2005; 103: 1457-1467.

7. Ellison LF, Pogany L, Mery LS. Childhood and adolescent cancer survival: a period analysis of data from the Canadian Cancer Registry. Eur J Cancer 2007; 43: 1967-1975.

8. Stiller CA. International patterns of cancer incidence in adolescents. Cancer Treat Rev 2007; 33: 631-645.

9. Barr R. On cancer control and the adolescent. Med Pediatr Oncol 1999; 32: 404-410.

10. Smith MA, Gurney JG, Ries LAG. Cancer among adolescents 15-19 years old. SEER Pediatric Monograph 1999: 157-164.

11. Albritton K, Bleyer WA. The management of cancer in the older adolescent. Eur J Cancer 2003; 39: 2584-2599.

12. Yeager ND, Hoshaw-Woodard S, Ruymann FB, Termuhlen A. Patterns of care among adolescents with malignancy in Ohio. J Pediatr Hematol Oncol 2006; 28: 17-22.

13. Klein-Geltink J, Shaw AK, Morrison HI, Barr RD, Greenberg ML. Use of paediatric versus adult oncology treatment centres by ado- lescents 15-19 years old: the Canadian Childhood Cancer Surveillance and Control Program. Eur J Cancer 2005; 41: 404-410.

14. Desandes E. Survival from adolescent cancer. Cancer Treatment Reviews 2007; 33: 609-615. 
15. Stock W, La M, Sanford B, et al; Children's Cancer Group; Cancer and Leukemia Group B. What determines the outcomes for adolescents and young adults with acute lymphoblastic leukemia treated on cooperative group protocols? A comparison of Children's Cancer Group and Cancer and Leukemia Group B studies. Blood 2008;112: 1646-1654

16. Tai E, Buchanan N, Westervelt L, Elimam D, Lawvere S. Treatment setting, clinical trial enrollment, and subsequent outcomes in adolescents with cancer: a literature review. Pediatrics 2014; 133: S91-S97.

17. Testi AM, Valsecchi MG, Conter V, et al. Difference in outcome of adolescents with acute lymphoblastic leukemia (ALL) enrolled in pediatric (AIEOP) and adult (GIMEMA) protocols. [Abstract] Blood 2004; 104: A-1954.

18. Boissel N, Auclerc MF, Lhéritier V, et al. Should adolescents with acute lympho- blastic leukemia be treated as old children or young adults? Comparison of the French FRALLE-93 and LALA-94 trials. J Clin Oncol 2003; 21: 774-780.

19. Paulussen M, Ahrens S, Juergens HF. Cure rates in Ewing tumor patients aged over 15 years are better in pediatric oncology units: Results of GPOH CESS/EICESS studies [abstract]. Proc Am Soc Clin Oncol 2003; 22: 3279.
20. Ferrari A, Thomas D, Franklin ARK, et al. Starting an adolescent and young adult program: some success stories and some obstacles to overcome. J Clin Oncol 2010; 28: 4850-4857.

21. Ferrari A, Clerici CA, Casanova M, et al. The youth project at the Istituto Nazionale Tumori in Milan. Tumori 2012; 98 : 399-407.

22. Stiller CA, Desandes E, Danon SE, et al. Cancer incidence and survival in European adolescents (1978-1997). Report from the Automated Childhood Cancer Information System project. Eur J Cancer 2006; 42: 2006-2018.

23. Tebbi CK. Treatment compliance in childhood and adolescence. Cancer 1993; 71(Suppl. 10): 3441-3449.

24. Kyngas HA, Kroll T, Duffy ME. Compliance in adolescents with chronic diseases: a review. J Adolesc Med 2000; 26: 379-388.

25. Morse JM, Mitcham C, Hupcey JE, Tason MC. Criteria for concept evaluation. J Adv Nurs 1996; 24: 385-390.

26. Barr RD. The adolescent with cancer. Eur J Cancer 2001; 37: $1523-1530$. 